\title{
On Certain Classes of Harmonic $p$-Valent Functions Defined by an Integral Operator
}

\author{
T. M. Seoudy \\ Department of Mathematics, Faculty of Science, Fayoum University, Fayoum 63514, Egypt \\ Correspondence should be addressed to T. M. Seoudy; tms00@fayoum.edu.eg \\ Received 3 November 2012; Accepted 18 December 2012 \\ Academic Editor: Frédéric Robert
}

Copyright (c) 2013 T. M. Seoudy. This is an open access article distributed under the Creative Commons Attribution License, which permits unrestricted use, distribution, and reproduction in any medium, provided the original work is properly cited.

We obtain coefficient characterization, extreme points, and distortion bounds of certain classes of harmonic $p$-valent functions defined by an integral operator.

\section{Introduction}

A continuous complex-valued function $f=u+i v$ defined in a simply connected complex domain $D$ is said to be harmonic in $D$ if both $u$ and $v$ are real harmonic in $D$. In any simply connected domain, we can write

$$
f=h+\bar{g}
$$

where $h$ and $g$ are analytic in $D$. We call $h$ the analytic part and $g$ the coanalytic part of $f$. A necessary and sufficient condition for $f$ to be locally univalent and sense preserving in $D$ is that $\left|h^{\prime}(z)\right|>\left|g^{\prime}(z)\right|$ in $D$ (see [1]).

Denote by $S_{H}$ the class of functions $f$ of the form (1) that are harmonic univalent and sense preserving in the unit disc $U=\{z:|z|<1\}$ for which $f(0)=f_{z}(0)-1=0$.

Recently, Jahangiri and Ahuja [2] defined the class $\mathscr{H}_{p}(p \in \mathbb{N}=\{1,2,3, \ldots\})$, consisting of all $p$-valent harmonic functions $f=h+\bar{g}$ that are sense preserving in $U$ and $h$, and $g$ are of the form

$$
h(z)=z^{p}+\sum_{k=p+1}^{\infty} a_{k} z^{k}, \quad g(z)=\sum_{k=p}^{\infty} b_{k} z^{k}, \quad\left|b_{p}\right|<1 .
$$

For $f=h+\bar{g}$ given by (2), we define the modified $p$ valent Salagean integral operator $I_{p, \lambda}^{n}$ of $f$ (see [3] and also [4] when $p=1$ ) as follows:

$$
I_{p, \lambda}^{n} f(z)=I_{p, \lambda}^{n} h(z)+(-1)^{n} \overline{I_{p, \lambda}^{n} g(z)},
$$

where

$$
\begin{aligned}
I_{p, \lambda}^{n} h(z)= & z^{p}+\sum_{k=p+1}^{\infty}\left(\frac{p}{p+\lambda(k-p)}\right)^{n} a_{k} z^{k} \\
& \left(p \in \mathbb{N} ; \lambda>0 ; n \in \mathbb{N}_{0}=\mathbb{N} \cup\{0\}\right), \\
I_{p, \lambda}^{n} g(z)= & \sum_{k=p}^{\infty}\left(\frac{p}{p+\lambda(k-p)}\right)^{n} b_{k} z^{k} \\
& \left(p \in \mathbb{N} ; \lambda>0 ; n \in \mathbb{N}_{0}\right) .
\end{aligned}
$$

For $p \in \mathbb{N}, \lambda>0, n \in \mathbb{N}_{0}, 0 \leq \alpha<1$, and $z \in U$, we let $\mathscr{H}_{p, \lambda}(n ; \alpha)$ denote the family of harmonic functions $f$ of the form (2) such that

$$
\operatorname{Re}\left\{\frac{I_{p, \lambda}^{n} f(z)}{I_{p, \lambda}^{n+1} f(z)}\right\}>\alpha,
$$

where $I_{p, \lambda}^{n} f$ is defined by (3).

We let the subclass $\mathscr{H}_{p, \lambda}^{-}(n ; \alpha)$ consists of harmonic functions $f_{n}=h+\bar{g}_{n}$ in $\mathscr{H}_{p, \lambda}(n ; \alpha)$ so that $h$ and $g_{n}$ are of the form

$$
h(z)=z^{p}-\sum_{k=p+1}^{\infty} a_{k} z^{k}, \quad g_{n}(z)=(-1)^{n} \sum_{k=p}^{\infty} b_{k} z^{k},
$$


We note that $\mathscr{H}_{p, 1}^{-}(n ; \alpha)=\mathscr{H}_{p}^{-}(n ; \alpha)$, where the class $\mathscr{H}_{p}^{-}(n ; \alpha)$ was defined and studied by Cotirla [5].

In this paper, we obtain coefficient characterization of the classes $\mathscr{H}_{p, \lambda}(n ; \alpha)$ and $\mathscr{H}_{p, \lambda}^{-}(n ; \alpha)$. We also obtain extreme points and distortion bounds for functions in the class $\mathscr{H}_{p, \lambda}^{-}(n ; \alpha)$.

\section{Coefficient Characterization}

Unless otherwise mentioned, we assume throughout this paper that $p \in \mathbb{N}, n \in \mathbb{N}_{0}, 0 \leq \alpha<1, a_{p}=1$, and $\lambda>0$. We begin with a sufficient condition for functions in $\mathscr{H}_{p, \lambda}(n ; \alpha)$.

Theorem 1. Let $f=h+\bar{g}$ so that $h$ and $g$ are given by (2). Furthermore, let

$$
\sum_{k=p}^{\infty}\left\{\Psi_{p, \lambda}(n, k, \alpha)\left|a_{k}\right|+\Phi_{p, \lambda}(n, k, \alpha)\left|b_{k}\right|\right\} \leq 2,
$$

where

$$
\begin{aligned}
\Psi_{p, \lambda}(n, k, \alpha)= & \left(\left(\frac{p}{p+\lambda(k-p)}\right)^{n}\right. \\
& \left.-\alpha\left(\frac{p}{p+\lambda(k-p)}\right)^{n+1}\right) \\
& \times(1-\alpha)^{-1}, \\
\Phi_{p, \lambda}(n, k, \alpha)= & \left(\left(\frac{p}{p+\lambda(k-p)}\right)^{n}\right. \\
& \times(1-\alpha)^{-1} .
\end{aligned}
$$

Then, $f$ is sense preserving in $U$ and $f \in \mathscr{H}_{p, \lambda}(n ; \alpha)$.

Proof. According to (2) and (3), we only need to show that

$$
\operatorname{Re}\left\{\frac{I_{p, \lambda}^{n} f(z)-\alpha I_{p, \lambda}^{n+1} f(z)}{I_{p, \lambda}^{n+1} f(z)}\right\} \geq 0 \quad(z \in U)
$$

It follows that

$$
\begin{aligned}
& \operatorname{Re}\left\{\frac{I_{p, \lambda}^{n} f(z)-\alpha I_{p, \lambda}^{n+1} f(z)}{I_{p, \lambda}^{n+1} f(z)}\right\} \\
& =\operatorname{Re}\left\{\left((1-\alpha) z^{p}\right.\right.
\end{aligned}
$$

$$
\begin{aligned}
+\sum_{k=p+1}^{\infty} \times & {\left[\left(\frac{p}{p+\lambda(k-p)}\right)^{n}\right.} \\
& \left.\left.-\alpha\left(\frac{p}{p+\lambda(k-p)}\right)^{n+1}\right] a_{k} z^{k}\right)
\end{aligned}
$$

$$
\begin{aligned}
& \times\left(z^{p}+\sum_{k=p+1}^{\infty}\left(\frac{p}{p+\lambda(k-p)}\right)^{n+1} a_{k} z^{k}\right. \\
& \left.+(-1)^{n+1} \sum_{k=p}^{\infty}\left(\frac{p}{p+\lambda(k-p)}\right)^{n+1} \bar{b}_{k} \bar{z}^{k}\right)^{-1}
\end{aligned}
$$$$
+\left(( - 1 ) ^ { n } \sum _ { k = p } ^ { \infty } \left[\left(\frac{p}{p+\lambda(k-p)}\right)^{n}\right.\right.
$$$$
\left.\left.+\alpha\left(\frac{p}{p+\lambda(k-p)}\right)^{n+1}\right] \bar{b}_{k} \bar{z}^{k}\right)
$$

$$
\begin{aligned}
\times\left(z^{p}+\sum_{k=p+1}^{\infty}\left(\frac{p}{p+\lambda(k-p)}\right)^{n+1} a_{k} z^{k}\right. \\
\left.\left.+(-1)^{n+1} \sum_{k=p}^{\infty}\left(\frac{p}{p+\lambda(k-p)}\right)^{n+1} \bar{b}_{k} \bar{z}^{k}\right)^{-1}\right\}
\end{aligned}
$$

$=\operatorname{Re}\{((1-\alpha)$

$$
\begin{aligned}
+\sum_{k=p+1}^{\infty} \times\left[\left(\frac{p}{p+\lambda(k-p)}\right)^{n}\right. \\
\left.\left.-\alpha\left(\frac{p}{p+\lambda(k-p)}\right)^{n+1}\right] a_{k} z^{k-p}\right)
\end{aligned}
$$

$$
\times\left(1+\sum_{k=p+1}^{\infty}\left(\frac{p}{p+\lambda(k-p)}\right)^{n+1} a_{k} z^{k-p}\right.
$$

$$
\begin{aligned}
& \left.+(-1)^{n+1} \sum_{k=p}^{\infty}\left(\frac{p}{p+\lambda(k-p)}\right)^{n+1} \bar{b}_{k} \bar{z}^{k} z^{-p}\right)^{-1} \\
& +\left(( - 1 ) ^ { n } \sum _ { k = p } ^ { \infty } \left[\left(\frac{p}{p+\lambda(k-p)}\right)^{n}\right.\right.
\end{aligned}
$$

$$
\begin{array}{r}
\left.+\alpha\left(\frac{p}{p+\lambda(k-p)}\right)^{n+1}\right] \\
\left.\times \bar{b}_{k} \bar{z}^{k} z^{-p}\right)
\end{array}
$$




$$
\begin{aligned}
\times(1 & +\sum_{k=p+1}^{\infty}\left(\frac{p}{p+\lambda(k-p)}\right)^{n+1} \\
& \times a_{k} z^{k-p}+(-1)^{n+1} \sum_{k=p}^{\infty}\left(\frac{p}{p+\lambda(k-p)}\right)^{n+1} \\
& \left.\left.\times \bar{b}_{k} \bar{z}^{k} z^{-p}\right)^{-1}\right\} \\
= & \operatorname{Re}\left\{\frac{1-\alpha+A(z)}{1+B(z)}\right\} .
\end{aligned}
$$

For $z=r e^{i \theta}$, we have

$$
\begin{aligned}
A\left(r e^{i \theta}\right)= & \sum_{k=p+1}^{\infty}\left[\left(\frac{p}{p+\lambda(k-p)}\right)^{n}\right. \\
& \left.-\alpha\left(\frac{p}{p+\lambda(k-p)}\right)^{n+1}\right] a_{k} r^{k-p} e^{i(k-p) \theta} \\
& +(-1)^{n} \sum_{k=p}^{\infty}\left[\left(\frac{p}{p+\lambda(k-p)}\right)^{n}\right. \\
& \left.\times \bar{b}_{k} r^{k-p} e^{-i(k+p) \theta}, \quad+\alpha\left(\frac{p}{p+\lambda(k-p)}\right)^{n+1}\right] \\
B\left(r e^{i \theta}\right)= & \sum_{k=p+1}^{\infty}\left(\frac{p}{p+\lambda(k-p)}\right)^{n+1} a_{k} r^{k-p} e^{i(k-p) \theta} \\
& +(-1)^{n+1} \sum_{k=p}^{\infty}\left(\frac{p}{p+\lambda(k-p)}\right)^{n+1} \bar{b}_{k} r^{k-p} e^{-i(k+p) \theta} .
\end{aligned}
$$

Setting that

$$
\frac{1-\alpha+A(z)}{1+B(z)}=(1-\alpha) \frac{1+w(z)}{1-w(z)}
$$

the proof will be complete if we can show that $|w(z)|<1$. Using the condition (7), we can write

$$
\begin{aligned}
|w(z)| & =\left|\frac{A(z)-(1-\alpha) B(z)}{A(z)+(1-\alpha) B(z)+2(1-\alpha)}\right| \\
& =\mid\left(\sum _ { k = p + 1 } ^ { \infty } \left[\left(\frac{p}{p+\lambda(k-p)}\right)^{n}\right.\right.
\end{aligned}
$$

$$
\begin{array}{r}
\left.\left.-\left(\frac{p}{p+\lambda(k-p)}\right)^{n+1}\right] a_{k} r^{k-p} e^{i(k-p) \theta}\right) \\
\times\left(2(1-\alpha)+\sum_{k=p+1}^{\infty} c_{k} a_{k} r^{k-p} e^{i(k-p) \theta}\right. \\
\left.+(-1)^{n} \sum_{k=p}^{\infty} d_{k} \bar{b}_{k} r^{k-p} e^{-i(k+p) \theta}\right)^{-1} \\
+\left(( - 1 ) ^ { n } \sum _ { k = p } ^ { \infty } \left[\left(\frac{p}{p+\lambda(k-p)}\right)^{n}\right.\right.
\end{array}
$$$$
\left.+\alpha\left(\frac{p}{p+\lambda(k-p)}\right)^{n+1}\right]
$$

$$
\begin{gathered}
\left.\times \bar{b}_{k} r^{k-p} e^{-i(k+p) \theta}\right) \\
\times\left(2(1-\alpha)+\sum_{k=p+1}^{\infty} c_{k} a_{k} r^{k-p} e^{i(k-p) \theta}\right. \\
\left.+(-1)^{n} \sum_{k=p}^{\infty} d_{k} \bar{b}_{k} r^{k-p} e^{-i(k+p) \theta}\right)^{-1} \mid \\
\leq \mid\left(\sum _ { k = p + 1 } ^ { \infty } \left[\left(\frac{p}{p+\lambda(k-p)}\right)^{n}\right.\right. \\
\left.\left.-\left(\frac{p}{p+\lambda(k-p)}\right)^{n+1}\right]\left|a_{k}\right| r^{k-p}\right)
\end{gathered}
$$$$
\times\left(2(1-\alpha)-\sum_{k=p+1}^{\infty} c_{k}\left|a_{k}\right| r^{k-p}\right.
$$$$
\left.-\sum_{k=p}^{\infty} d_{k}\left|b_{k}\right| r^{k-p}\right)^{-1}
$$$$
+\left(\sum _ { k = p } ^ { \infty } \left[\left(\frac{p}{p+\lambda(k-p)}\right)^{n}\right.\right.
$$

$$
\left.\left.+\left(\frac{p}{p+\lambda(k-p)}\right)^{n+1}\right]\left|b_{k}\right| r^{k-p}\right)
$$$$
\times\left(2(1-\alpha)-\sum_{k=p+1}^{\infty} c_{k}\left|a_{k}\right| r^{k-p}\right.
$$$$
\left.-\sum_{k=p}^{\infty} d_{k}\left|b_{k}\right| r^{k-p}\right)^{-1} \mid
$$

$=\mid\left(\sum_{k=p+1}^{\infty}\left[\left(\frac{p}{p+\lambda(k-p)}\right)^{n}\right.\right.$ 


$$
\begin{gathered}
\left.\left.\quad-\left(\frac{p}{p+\lambda(k-p)}\right)^{n+1}\right]\left|a_{k}\right| r^{k-p}\right) \\
\times\left(4(1-\alpha)-\sum_{k=p}^{\infty}\left\{c_{k}\left|a_{k}\right|+d_{k}\left|b_{k}\right|\right\} r^{k-p}\right)^{-1} \\
+\left(\sum _ { k = p } ^ { \infty } \left[\left(\frac{p}{p+\lambda(k-p)}\right)^{n}\right.\right. \\
\left.\left.\quad+\left(\frac{p}{p+\lambda(k-p)}\right)^{n+1}\right]\left|b_{k}\right| r^{k-p}\right)^{-1} \mid \\
\times\left(4(1-\alpha)-\sum_{k=p}^{\infty}\left\{c_{k}\left|a_{k}\right|+d_{k}\left|b_{k}\right|\right\} r^{k-p}\right)^{n} \\
<\mid\left(\sum _ { k = p + 1 } ^ { \infty } \left[\left(\frac{p}{p+\lambda(k-p)}\right)^{n}\right.\right. \\
\left.\left.\left.\times\left(4(1-\alpha)-\sum_{k=p}^{\infty}\left\{c_{k}\left|a_{k}\right|+d_{k}\left|b_{k}\right|\right\}\right)^{-1} \mid \frac{p}{p+\lambda(k-p)}\right)^{n+1}\right]\left|b_{k}\right|\right) \\
+\left(\sum_{k=p}^{\infty}\left[\left(\frac{p}{p+\lambda(k-p)}\right)^{n+1}\right]\left|a_{k}\right|\right) \\
\times\left(4(1-\alpha)-\sum_{k=p}^{\infty}\left\{c_{k}\left|a_{k}\right|+d_{k}\left|b_{k}\right|\right\}\right)^{-1} \\
+\left(\frac{p-p)}{p(k-1}\right)
\end{gathered}
$$

where

$$
\begin{aligned}
& c_{k}=\left(\frac{p}{p+\lambda(k-p)}\right)^{n}+(1-2 \alpha)\left(\frac{p}{p+\lambda(k-p)}\right)^{n+1}, \\
& d_{k}=\left(\frac{p}{p+\lambda(k-p)}\right)^{n}-(1-2 \alpha)\left(\frac{p}{p+\lambda(k-p)}\right)^{n+1} .
\end{aligned}
$$

The harmonic functions are as follows:

$$
f(z)=z^{p}+\sum_{k=p+1}^{\infty} \frac{1}{\Psi_{p, \lambda}(n, k, \alpha)} x_{k} z^{k}
$$

$$
+\sum_{k=p}^{\infty} \frac{1}{\Phi_{p, \lambda}(n, k, \alpha)} \overline{y_{k} z^{k}}
$$

where $\sum_{k=p+1}^{\infty}\left|x_{k}\right|+\sum_{k=p}^{\infty}\left|y_{k}\right|=1$ show that the coefficient bound given by (7) is sharp. The functions of the form (8) are in the class $\mathscr{H}_{p, \lambda}(n ; \alpha)$ because

$$
\begin{gathered}
\sum_{k=p}^{\infty}\left\{\Psi_{p, \lambda}(n, k, \alpha)\left|a_{k}\right|+\Phi_{p, \lambda}(n, k, \alpha)\left|b_{k}\right|\right\} \\
=1+\sum_{k=p+1}^{\infty}\left|x_{k}\right|+\sum_{k=p}^{\infty}\left|y_{k}\right|=2 .
\end{gathered}
$$

This completes the proof of Theorem 1.

In the following theorem, it is shown that the condition (7) is also necessary for functions $f_{n}=h+\bar{g}_{n}$, where $h$ and $g_{n}$ are of the form (6).

Theorem 2. Let $f_{n}=h+\bar{g}_{n}$, where $h$ and $g_{n}$ are given by (6). Then, $f_{n} \in \mathscr{H}_{p, \lambda}^{-}(n ; \alpha)$ if and only if

$$
\sum_{k=p}^{\infty}\left\{\Psi_{p, \lambda}(n, k, \alpha) a_{k}+\Phi_{p, \lambda}(n, k, \alpha) b_{k}\right\} \leq 2,
$$

where $\Psi_{p, \lambda}(n, k, \alpha)$ and $\Phi_{p, \lambda}(n, k, \alpha)$ are given by (8) and (9), respectively.

Proof. Since $\mathscr{H}_{p, \lambda}^{-}(n ; \alpha) \subset \mathscr{H}_{p, \lambda}(n ; \alpha)$, we only need to prove the "only if" part of the theorem. To this end, for functions $f_{n}=h+\bar{g}_{n}$, where $h$ and $g_{n}$ are given by (6), we notice that the condition $\operatorname{Re}\left\{I_{p, \lambda}^{n} f(z) / I_{p, \lambda}^{n+1} f(z)\right\}>\alpha$ is equivalent to

$$
\operatorname{Re}\left\{\left((1-\alpha) z^{p}\right.\right.
$$

$$
\begin{aligned}
&-\sum_{k=p+1}^{\infty} \times {\left[\left(\frac{p}{p+\lambda(k-p)}\right)^{n}\right.} \\
&\left.\left.-\alpha\left(\frac{p}{p+\lambda(k-p)}\right)^{n+1}\right] a_{k} z^{k}\right)
\end{aligned}
$$

$$
\times\left(z^{p}-\sum_{k=p+1}^{\infty}\left(\frac{p}{p+\lambda(k-p)}\right)^{n+1} a_{k} z^{k}\right.
$$

$$
\left.+(-1)^{2 n} \sum_{k=p}^{\infty}\left(\frac{p}{p+\lambda(k-p)}\right)^{n+1} b_{k} \bar{z}^{k}\right)^{-1}
$$




$$
\left.\begin{array}{l}
+(-1)^{2 n-1} \sum_{k=p}^{\infty} \\
\times\left[\left(\frac{p}{p+\lambda(k-p)}\right)^{n}\right. \\
\left.\left.+\alpha\left(\frac{p}{p+\lambda(k-p)}\right)^{n+1}\right] b_{k} \bar{z}^{k}\right) \\
\times\left(z^{p}-\sum_{k=p+1}^{\infty}\left(\frac{p}{p+\lambda(k-p)}\right)^{n+1} a_{k} z^{k}\right. \\
\left.+(-1)^{2 n} \sum_{k=p}^{\infty}\left(\frac{p}{p+\lambda(k-p)}\right)^{n+1} \bar{b}_{k} \bar{z}^{k}\right)^{-1}
\end{array}\right\}
$$

The previous required condition (19) must hold for all values of $z$ in $U$. Upon choosing the values of $z$ on the positive real axis where $0 \leq z=r<1$, we must have

$$
\begin{aligned}
(1-\alpha)- & \sum_{k=p+1}^{\infty}\left[\left(\frac{p}{p+\lambda(k-p)}\right)^{n}\right. \\
& \left.\left.-\alpha\left(\frac{p}{p+\lambda(k-p)}\right)^{n+1}\right] a_{k} r^{k-p}\right) \\
\times & \left(1-\sum_{k=p+1}^{\infty}\left(\frac{p}{p+\lambda(k-p)}\right)^{n+1} a_{k} r^{k-p}\right. \\
+ & +\sum_{k=p}^{\infty}\left[\left(\frac{p}{p+\lambda(k-p)}\right)^{n+1} b_{k} r^{k-p}\right)^{-1} \\
& +\sum_{k=p}^{\infty}\left(\frac{p}{p+\lambda(k-p)}\right)^{n} \\
\times & \left(1-\sum_{k=p+1}^{\infty}\left(\frac{p}{p+\lambda(k-p)}\right)^{n+1} a_{k} r^{k-p} b_{k} r^{k-p}\right)^{-1} \\
& \left.+\alpha\left(\frac{p}{p+\lambda(k-p)}\right)^{n+1}\right] \\
& \left.+b_{k} r^{k-p}\right)
\end{aligned}
$$

If the condition (18) does not hold, then the numerator in (20) is negative for $r$ sufficiently close to 1 . Hence there exists $z_{0}=r_{0}$ in $(0,1)$ for which the quotient in $(20)$ is negative. This contradicts the required condition for $f_{n} \in \mathscr{H}_{p, \lambda}^{-}(n ; \alpha)$, and so the proof of Theorem 2 is completed.

\section{Extreme Points and Distortion Theorem}

Our next theorem is on the extreme points of convex hulls of the class $\mathscr{H}_{p, \lambda}^{-}(n ; \alpha)$ denoted by $c l c o \mathscr{H}_{p, \lambda}^{-}(n ; \alpha)$.

Theorem 3. Let $f_{n}=h+\bar{g}_{n}$, where $h$ and $g_{n}$ are given by (6). Then, $f_{n} \in \mathscr{H}_{p, \lambda}^{-}(n ; \alpha)$ if and only if

$$
f_{n}(z)=\sum_{k=p}^{\infty}\left[x_{k} h_{k}(z)+y_{k} g_{n_{k}}(z)\right]
$$

where

$$
h_{1}(z)=z^{p}, \quad h_{k}(z)=z^{p}-\frac{1}{\Psi_{p, \lambda}(n, k, \alpha)} z^{k}
$$

$$
(k=p+1, p+2, p+3, \ldots) \text {, }
$$

$$
\begin{gathered}
g_{n_{k}}(z)=z^{p}+(-1)^{n-1} \frac{1}{\Phi_{p, \lambda}(n, k, \alpha)} \bar{z}^{k} \\
(k=p, p+1, p+2, \ldots), \\
x_{k}, y_{k} \geq 0, \quad x_{p}=1-\sum_{k=p+1}^{\infty} x_{k}-\sum_{k=p}^{\infty} y_{k} .
\end{gathered}
$$

In particular, the extreme points of the class $\mathscr{H}_{p, \lambda}^{-}(n ; \alpha)$ are $\left\{h_{k}\right\}$ and $\left\{g_{n_{k}}\right\}$.

Proof. Suppose that

$$
\begin{aligned}
f_{n}(z)= & \sum_{k=p}^{\infty}\left(x_{k} h_{k}(z)+y_{k} g_{n_{k}}(z)\right) \\
= & \sum_{k=p}^{\infty}\left(x_{k}+y_{k}\right) z^{p}-\sum_{k=p+1}^{\infty} \frac{1}{\Psi_{p, \lambda}(n, k, \alpha)} x_{k} z^{k} \\
& +(-1)^{n-1} \sum_{k=1}^{\infty} \frac{1}{\Phi_{p, \lambda}(n, k, \alpha)} y_{k} \bar{z}^{k} .
\end{aligned}
$$

Then,

$$
\begin{aligned}
& \sum_{k=p+1}^{\infty} \Psi_{p, \lambda}(n, k, \alpha)\left(\frac{1}{\Psi_{p, \lambda}(n, k, \alpha)} x_{k}\right) \\
& \quad+\sum_{k=1}^{\infty} \Phi_{p, \lambda}(n, k, \alpha)\left(\frac{1}{\Phi_{p, \lambda}(n, k, \alpha)} y_{k}\right) \\
& =\sum_{k=p+1}^{\infty} x_{k}+\sum_{k=p}^{\infty} y_{k}=1-x_{p} \leq 1,
\end{aligned}
$$

and so $f_{n} \in \operatorname{clco} \mathscr{H}_{p, \lambda}^{-}(n ; \alpha)$. 
Conversely, if $f_{n} \in \operatorname{clco} \mathscr{H}_{p, \lambda}^{-}(n ; \alpha)$, then

$$
a_{k} \leq \frac{1}{\Psi_{p, \lambda}(n, k, \alpha)}, \quad b_{k} \leq \frac{1}{\Phi_{p, \lambda}(n, k, \alpha)}
$$

Set that

$$
\begin{array}{ll}
x_{k}=\Psi_{p, \lambda}(n, k, \alpha) a_{k} & (k=p+1, p+2, p+3, \ldots), \\
y_{k}=\Phi_{p, \lambda}(n, k, \alpha) b_{k} \quad(k=p, p+1, p+2, \ldots) .
\end{array}
$$

Then note that by Theorem $2,0 \leq x_{k} \leq 1,(k=p+1, p+$ $2, p+3, \ldots)$, and $0 \leq y_{k} \leq 1,(k=p, p+1, p+2, \ldots)$. We define that $x_{p}=1-\sum_{k=p+1}^{\infty} x_{k}-\sum_{k=p}^{\infty} y_{k}$ and note that by Theorem 2, $x_{p} \geq 0$. Consequently, we obtain $f_{n}(z)=$ $\sum_{k=p}^{\infty}\left\{x_{k} h_{k}(z)+y_{k} g_{k}(z)\right\}$ as required.

The following theorem gives the distortion bounds for functions in the class $\mathscr{H}_{p, \lambda}^{-}(n ; \alpha)$ which yields a covering result for this class.

Theorem 4. Let $f_{n}(z) \in \mathscr{H}_{p, \lambda}^{-}(n ; \alpha)$. Then, for $|z|=r<1$, we have

$$
\begin{array}{r}
\left(1-b_{p}\right) r^{p}-\left\{\Gamma_{p, \lambda}(n, \alpha)-\Delta_{p, \lambda}(n, \alpha)\right\} r^{p+1} \leq\left|f_{n}(z)\right| \\
\leq\left(1+b_{p}\right) r^{p}+\left\{\Gamma_{p, \lambda}(n, \alpha)-\Delta_{p, \lambda}(n, \alpha) b_{p}\right\} r^{p+1},
\end{array}
$$

where

$$
\begin{aligned}
\Gamma_{p, \lambda}(n, \alpha) & =\frac{1-\alpha}{(p /(p+\lambda))^{n}-\alpha(p /(p+\lambda))^{n+1}} \\
\Delta_{p, \lambda}(n, \alpha) & =\frac{1+\alpha}{(p /(p+\lambda))^{n}-\alpha(p /(p+\lambda))^{n+1}} .
\end{aligned}
$$

The result is sharp.

Proof. We only prove the right-hand inequality. The proof for the left-hand inequality is similar and will be omitted. Let $f_{n}(z) \in \mathscr{H}_{p, \lambda}^{-}(n ; \alpha)$. Taking the absolute value of $f_{n}$, we have

$$
\begin{aligned}
\left|f_{n}(z)\right| \leq & \left(1+b_{p}\right) r^{p}+\sum_{k=p+1}^{\infty}\left(a_{k}+b_{k}\right) r^{k} \\
\leq & \left(1+b_{p}\right) r^{p}+\sum_{k=p+1}^{\infty}\left(a_{k}+b_{k}\right) r^{p+1} \\
= & \left(1+b_{p}\right) r^{p}+\Gamma_{p, \lambda}(n, \alpha) \\
& \times \sum_{k=p+1}^{\infty} \frac{1}{\Gamma_{p, \lambda}(n, \alpha)}\left(a_{k}+b_{k}\right) r^{p+1} \\
\leq & \left(1+b_{p}\right) r^{p}+\Gamma_{p, \lambda}(n, \alpha) r^{p+1}
\end{aligned}
$$

$$
\begin{aligned}
& \times \sum_{k=p+1}^{\infty}\left\{\Psi_{p, \lambda}(n, k, \alpha) a_{k}+\Phi_{p, \lambda}(n, k, \alpha) b_{k}\right\} \\
\leq & \left(1+b_{p}\right) r^{p} \\
& +\left\{\Gamma_{p, \lambda}(n, k, \alpha)-\Delta_{p, \lambda}(n, k, \alpha) b_{p}\right\} r^{p+1} .
\end{aligned}
$$

The bounds given in Theorem 4 for functions $f_{n}=h+\bar{g}_{n}$, whereh and $g_{n}$ of form (6), also hold for functions of form (2) if the coefficient condition (7) is satisfied. The upper bound given for $f \in \mathscr{H}_{p, \lambda}^{-}(n ; \alpha)$ is sharp, and the equality occurs for the functions

$$
\begin{aligned}
f(z)= & z^{p}+b_{p} \bar{z}^{p} \\
& -\left(\frac{1-\alpha}{(p /(p+\lambda))^{n}-\alpha(p /(p+\lambda))^{n+1}}\right. \\
& \left.\quad-\frac{1-\alpha}{(p /(p+\lambda))^{n}+\alpha(p /(p+\lambda))^{n+1}} b_{p}\right) \bar{z}^{p+1},
\end{aligned}
$$

$$
\begin{aligned}
f(z)= & z^{p}+b_{p} \bar{z}^{p} \\
& -\left(\frac{1-\alpha}{(p /(p+\lambda))^{n}-\alpha(p /(p+\lambda))^{n+1}}\right. \\
& \left.\quad-\frac{1-\alpha}{(p /(p+\lambda))^{n}+\alpha(p /(p+\lambda))^{n+1}} b_{p}\right) z^{p+1}
\end{aligned}
$$

showing that the bounds given in Theorem 4 are sharp.

Remark 5. (i) Putting $\lambda=1$ in the previous results, we obtain the results of Cotirla [5].

(ii) Putting $\lambda=1$ in the previous results, we obtain the results of Cotirla [6], when $\beta=0$.

\section{Acknowledgment}

The author is grateful to the referees for their valuable suggestions.

\section{References}

[1] J. Clunie and T. Sheil-Small, "Harmonic univalent functions," Annales Academice Scientiarum Fennice Mathematica A, vol. 9, p. 3-25, 1984.

[2] J. M. Jahangiri and O. P. Ahuja, "Multivalent harmonic starlike functions," Annales Mariae Curie-SkThlodowska A, vol. 56, pp. $1-13,2001$.

[3] M. K. Aouf, A. O. Mostafa, and R. El-Ashwah, "Sandwich theorems for $\mathrm{p}$-valent functions defined by a certain integral operator," Mathematical and Computer Modelling, vol. 53, no. 9-10, pp. 1647-1653, 2011.

[4] F. M. Al-Oboudi, "On univalent functions defined by a generalized Sălăgean operator," International Journal of Mathematics 
and Mathematical Sciences, vol. 2004, no. 27, pp. 1429-1436, 2004.

[5] L.-I. Cotirla, "Harmonic multivalent functions defined by integral operator," Studia Universitatis Babeş-Bolyai, vol. 54, no. 1, pp. 65-74, 2009.

[6] L.-I. Cotirla, "A new class of harmonic multivalent functions defined by an integral operator," Acta Universitatis Apulensis, vol. 21, pp. 55-63, 2010. 


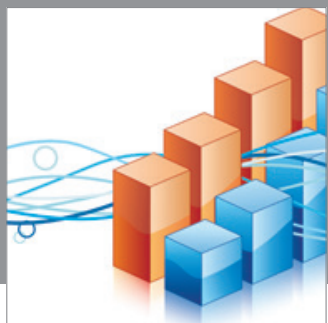

Advances in

Operations Research

mansans

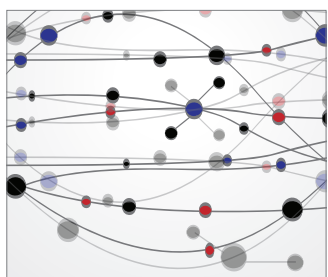

The Scientific World Journal
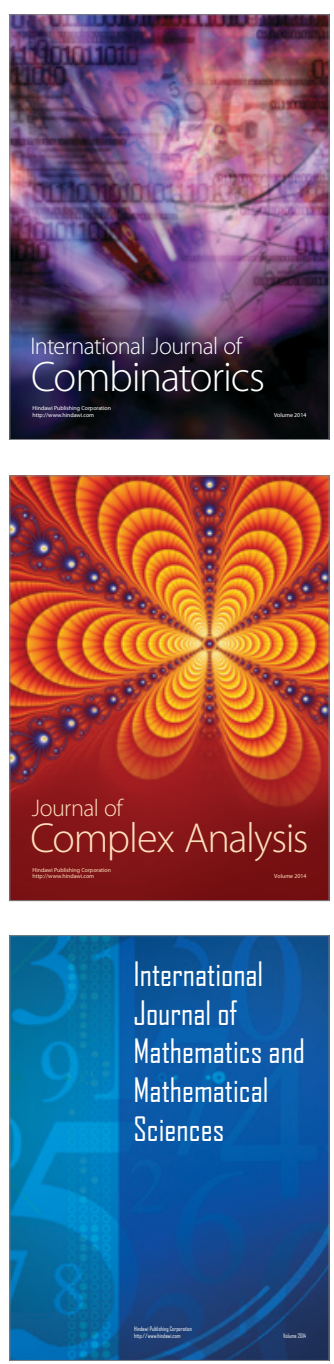
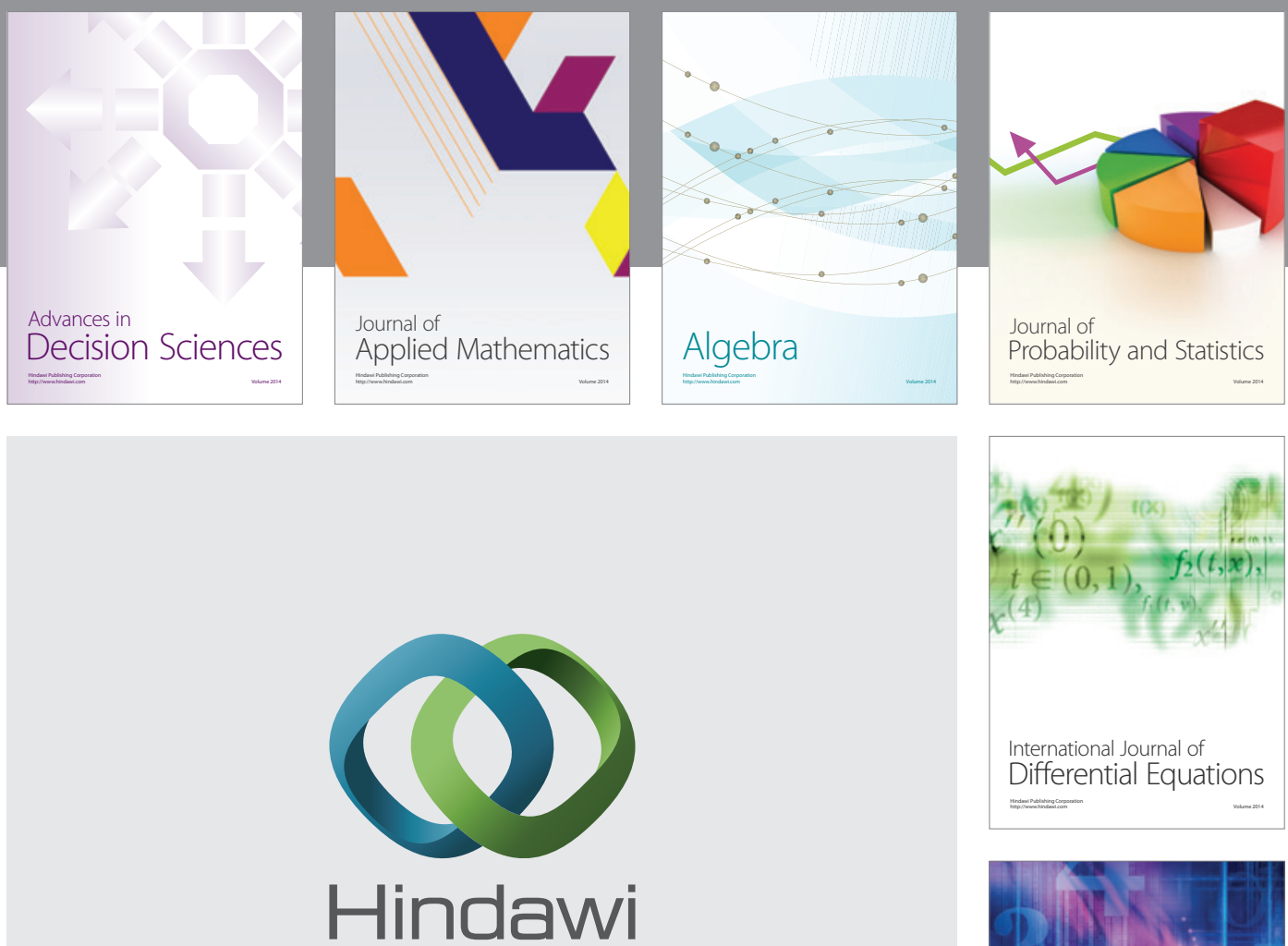

Submit your manuscripts at http://www.hindawi.com
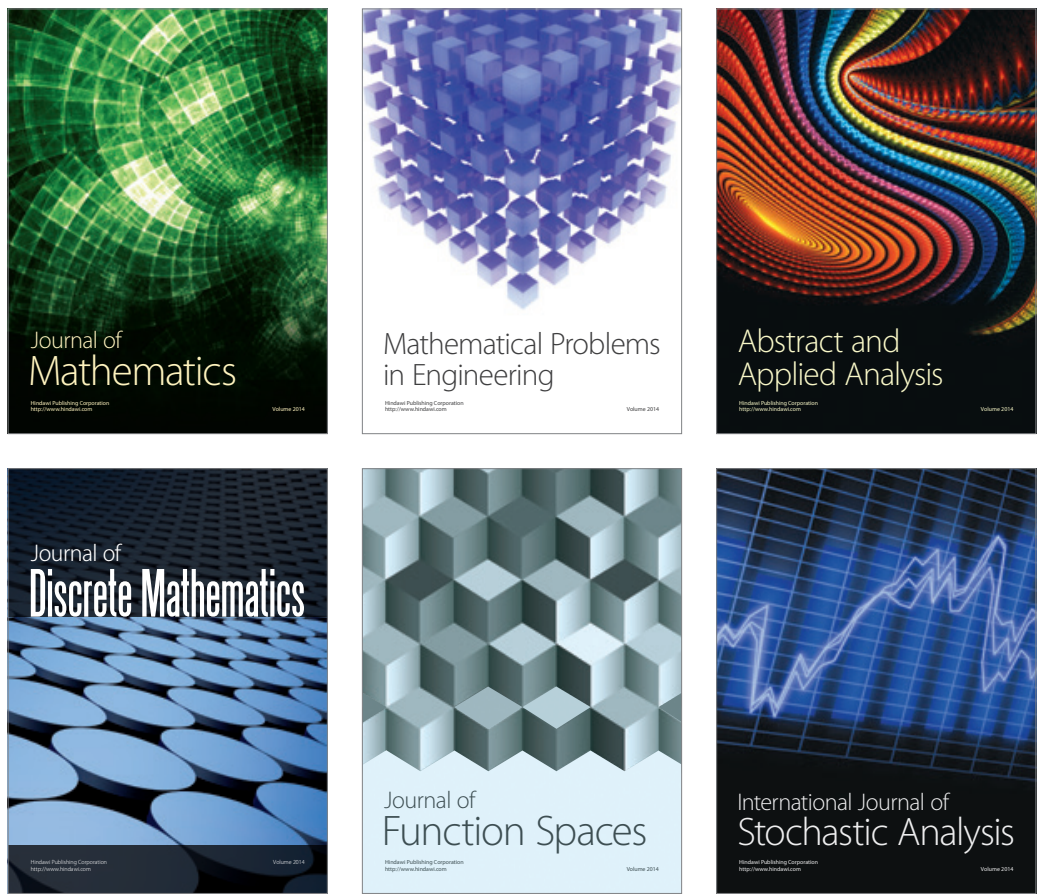

Journal of

Function Spaces

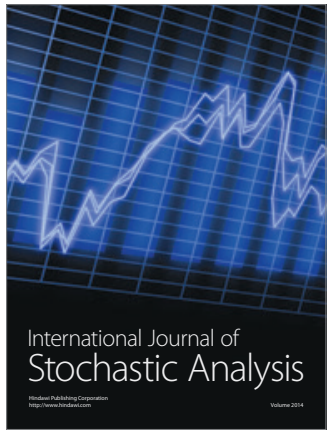

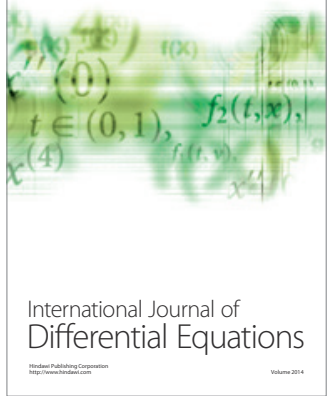
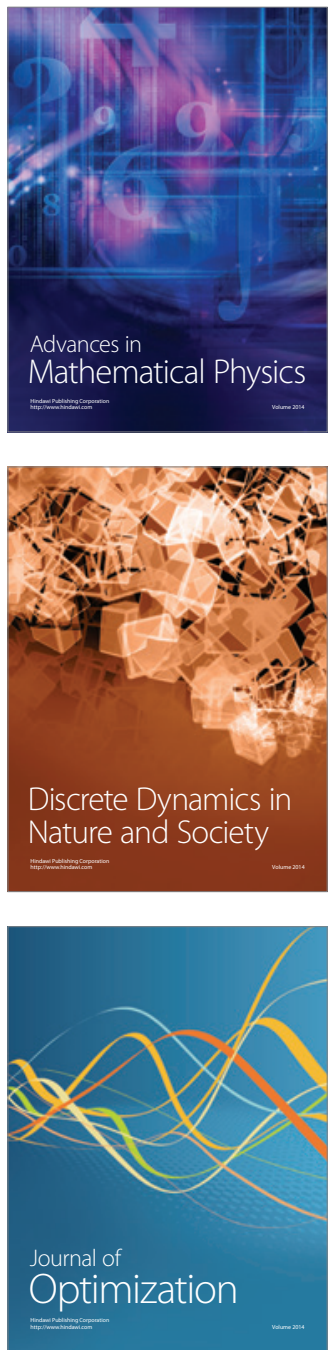